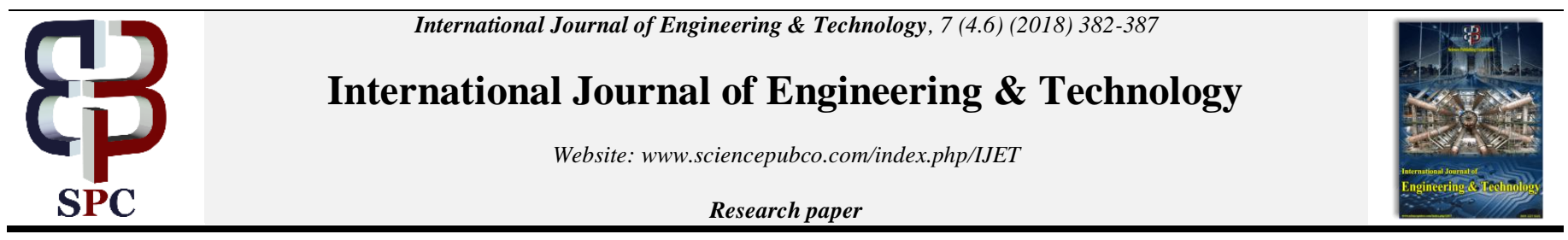

\title{
Cross Layer Design based Hybrid Error Control for Packet Aggregation over WSN
}

\author{
Lakshmi. $S^{1}$, Dr. Selvakumar raja.S ${ }^{2}$ \\ ${ }^{1}$ Department of Electronics and Communication -SCSVMV University, Enathur, Kanchipuram-631501, ${ }^{2}$ Department of Electronics and \\ Communication- Kakatiya Institute of Technology \& Science for Women, Nizamabad, Telungana-503003 \\ *Corresponding author E-mail: msklakshmi@gmail.com,
}

\begin{abstract}
In general sensor networks have a wide range of application and also play a major role in developing precision and timely information. Mostly it deals with real world applications and in many cases the data sensed by the nodes should deliver within the time constriction. Meeting the deadlines is mandate for the applications and the data should be processed as soon as possible and without major data losses. The objective of this scheme is to collect data with high accuracy and low latency. Collecting and processing the data by means of aggregation will greatly reduce the congestion data rate. Cross Layer Design based Hybrid Error Control (CLDHEC) mechanism is proposed for reducing error rate occurred in sensor network which includes formation of network, data aggregation and transmission. Error control technique named Adaptive Forward Error Correction is used to defend the video transmission by recovering source information losses. FEC mechanism along with packet range control greatly increases the FEC efficiency in wireless networks. The service quality for different kind of data can be improved by lessening error rate using adaptive error control mechanism with cross layered design during packet aggregation.
\end{abstract}

\section{Keywords: Error control, Erroneous block, FEC, Packet range control, Sensor networks}

\section{Introduction:}

WSN includes numerous tiny sensor nodes (or sensors for short) that is deployed over sensing environment for the purpose of tracking and monitoring. In recent years multimedia streaming applications have been emerged in wide area and become as prominent eye catching method. However maintaining multimedia streaming applications is difficult due to low bandwidth resources, low link quality, delay in routing information therefore video/image compressing increases the resources of networks. Detected information is combined in periodic levels, accumulated "innetwork" at sink hub or node which may themselves be more extravagant in capabilities and resources. Information are then conveyed to the destination either through on-request message of sink or the base station; higher request node.

Types of error control techniques

i). FEC is a prominent method for error control which employs error correcting codes for correcting the errors occurred due to channel defections. Redundancy bit is added (parity s-bits) to the information packets before they are transmitted and the bit size may vary with accordance to the packet size. The $\square$ redundancy is used by the receiver for error detection and correction purpose. Various FEC codes are optimized for specific packet sizes, channel requirements and conditions, and reliability such as linear block codes, ReedSolomon (RS) codes and Convolutional codes.

ii). Automatic Repeat Request (ARQ) is an error detection mechanism hence only detection is provided and not able to correct any packets received with error; therefore the eroded packets are requested to retransmit instead.

iii). Low-Density Parity-Check (LDPC) codes.

iv). Joint source codes.

Fundamentally FEC is enhanced for increasing the video throughput at receiver end before FEC recovering process and as needs be an equivalent or better mistake recovery execution is accomplished with less data transfer capacity utilization. Accordingly, an adaptive FEC scheme joined with packet size control is developed to simultaneously adjust the transmitting packet size and the level of FEC excess dependent on the minimum data transfer capacity utilization strategy. The FEC code with various scales such as bitlevel, byte-level and packet level affects the error recovery execution over the blunder inclined channel. By viewing bundles as the FEC coding things and marking a succession number onto every packet to show the relating position inside the square, packet level FEC is appeared to have a high proficiency over error-prone remote systems [1]. 


\section{Related Works}

Numerous research works have been carried out for error recovery and to produce better throughput at the receiver end. Cross-layer analysis of error control schemes [2] was proposed in which FEC coding significantly progresses the error resiliency with the help of adding redundant bits through the channel. The improvement can be exploited by transmitting power control or extending intermediary nodes through channel-aware geographical routing protocols in sensor networks. The consequences of their investigation uncover that for hybrid ARQ plans and certain FEC codes, the bounce length augmentation diminishes both the vitality utilization and the conclusion to end inertness subject to an objective parcel blunder rate (PER) contrasted with ARQ. They likewise demonstrate that the upsides of FEC codes are considerably more articulated as the system thickness increments. Conversely, transmit power control results in significant savings in energy consumption at the cost of increased latency for certain FEC codes [3]. Since transmit control expands the quantity of bounces, this system presents a noteworthy increment in inactivity, which is an exchange off for the FEC codes.

Cross layer examination adjust steering, physical layers, and MAC which are begun. The cross layer examination encourage a far reaching appraisal of programmed rehash ask for ARQ, FEC and half and half ARQ strategies in WSN [4]. SCoRe1 [5] was proposed for exact estimate of expansive genuine sensor organize motions through the accumulation of a little part of information focuses. SCoRe1 contains assorted insertion methods, either deterministic or probabilistic, and implants a control plan to routinely adjust the recuperation conduct with intermittent flag variety insights, while jumping the reconstruction error. With circuit switching [6], a biterror rate suitable for real-time applications such as voice and video is guaranteed by adopting robust FEC codes and proper powerbudget margins to face fading problems. With this approach, ARQ is used only for applications that necessitate a much lower error rate and can tolerate high delays. Packet technique that introduced in the error recovery uses ARQ even for real-time traffic and hence packet transmission time increases. A scheduling scheme for hybrid FEC/ARQ error control [7] was proposed, here both the data packets and FEC packets significance are evaluated by considering factors like format structure of data dependency, scalable video, packets transmission history as well as the deadline of decoding packets. Therefore scheduling hybrid scheme can able to achieve more stable playback video quality by considering the advantage of both FEC and ARQ.

Hybrid application-layer error correction consisting of FEC and retransmissions is a mechanism proposed for reducing error rate at application layer level. Integrated joint source-channel coding framework [8] where error resilient source coding, channel coding, and error concealment are jointly considered in order to achieve the best video delivery quality. The sequential joint source channel approach where error resilient source coding and channel coding are not fully integrated. Due to variations in channel condition and inadequate resources the video transmission faces many challenges. A network coding/ARQ hybrid error-control scheme [9] was proposed for achieving good-put and reliable transmission. Adaptation of coded packets is allowed from the source to the estimated condition of channel.

Optimal Progressive Error Recovery Algorithm (OPERA) [10] was proposed for reducing total number of retransmissions. Here decodeable LDPC codes are used iteratively for complete decoding process. In OPERA, entity transitional sensors that relay data towards the base station which are decoded for reliable packet outcomes. The incoming packets are encoded/decoded while employing a progressive decrease in parity bits as data reaches the receiver end. On-off error control coding scheme [11] was proposed to reduce error rate at the receiver node. Packet loss reduction in feedback control systems is crucial since control systems greatly affected by external disturbances. To minimize tracking errors channel coding is applied in a controlled plant. Based on communication and control layers (link layer and transport layer) the On-Off Error Control Scheme (OOECS) is implemented. Lightweight Error Concealment (EC) scheme [12] to support QoS was proposed to maintain the acceptable quality of multimedia transmission in WMSN. Scalable high efficiency video coding is pertained to control the packet rate with differential Quantization Parameters (QPs). The volume of information gets increased when the count of sensor nodes increased, in such case the QoS gets affected due to surplus amount of packet drop. Therefore Error Concealment is applied to overcome the erroneous at multipath routing. Cooperative ARQ for Relay Selection (CARQ_RS) protocol [13] is developed by applying semi markov process for time related multipath fading channels. The relay is selected by updating relay policy and the relay selection scheme involves three models such as permanent, reactive and adaptive. Comparing the permanent selection method adaptive and reactive schemes gives better potential gain because of selecting the adaptive and reactive nodes frequently. MAC and physical layer with cooperative communication is used to reduce the packet error rate in the scheme named cross layer optimisation with cooperative communication [14]. Minimum power cost with precise PER is evaluated either single relay node or between two relay nodes.

\section{Proposed Work}

Cross Layer Design based Hybrid Error Control (CLDHEC) is proposed to reduce the data loss during transmission over source node to destination. Due to geographical changes; packet or signal loss occurs and it leads to catastrophic issues in real time applications. To overcome these issues active relay nodes are selected for data transmission and if any packet losses occur then it can be retransmitted through partially observable channel condition. Figure 1 describes about experimental setup for proposed scheme.

\section{Adaptive FEC with packet range control:}

FEC is an error correcting code used for controlling errors over unreliable channels. FEC uses redundant data at the receiver end for deducting the errors at some location over the path and correcting the errors without retransmission process. However the process can be carried only when the node has higher forward channel bandwidth. If the node to node communication has lower rate channel bandwidth requests for retransmission is essential. The packet range FEC is used to safeguard the video stream that improves the properties of wireless transmission. Integrating FEC redundancy with packet range control greatly achieves the throughput quality and minimizes bandwidth overhead.

Principally the source hub transmits an un-coded packet or a packet coded with a lower error amendment capacity to the destined hub that is passed over the neighbor hubs. In the event that the packet at the beneficiary end got with error, then the destined node replies with NACK message, a negative affirmation (acknowledgement) message to source hub. Later the packet is resent with higher error correction code. 


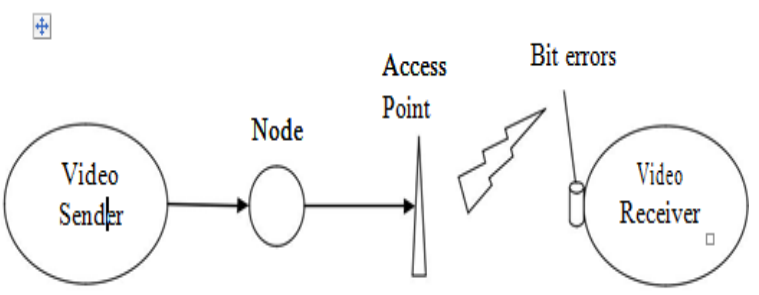

Fig.1: Experimental setup

In the packet level (long and short range) RS block (n, k) the transmitted block can be completely constructed at the receiver end by receiving ' $\mathrm{k}$ ' messages. The probability of successful recovery is given in equation (1),

$$
R=\sum_{i=0}^{h} n_{i} \times P_{p k t} i^{i} \times\left(1-P_{p k t}\right)^{n-i}
$$

Here $\mathrm{P}_{\mathrm{pkt}}$ is estimated amount for the ratio of corrupted packets to the total amount of transmitted packets. For getting reliable estimation the packets present in packet error rate need to be in same range or size. The average bit error rate $\mathrm{P}_{\mathrm{b}}$ in the channel is given by,

$$
\begin{aligned}
& P \\
& \text { plat }
\end{aligned}=1-\left(\begin{array}{c}
1-P)^{\tau} \\
b
\end{array}\right.
$$

Here' $t$ ' is referred to packet range or size measured in bits. By applying segmentation factor the packets can be divided into long range and short range. The collided packets can be recovered using Dynamic Collision Recovery (DCR) mechanism.

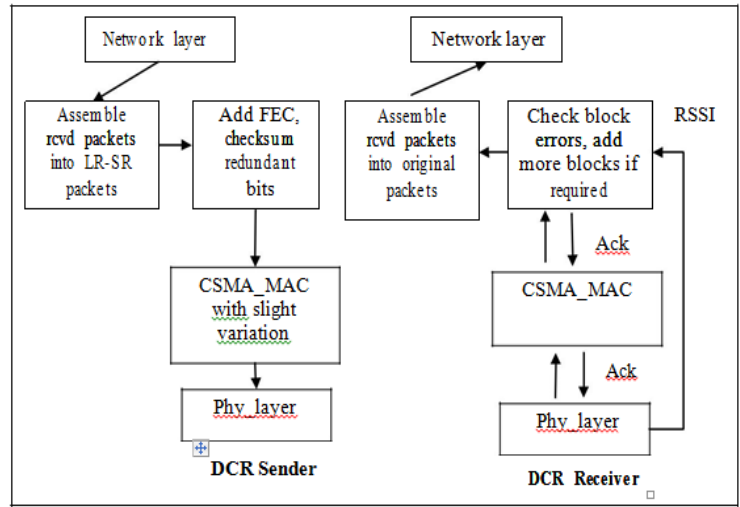

Fig.2: Dynamic recovery collision mechanism

Usually the sender hub converts the potential data collisions into LR_SR collisions and recuperates the packets that are corrupted from collision using this hybrid scheme. DCR mechanism is shown in the figure and protocol describes the proposed CLDHEC method to recuperate the packets which have been collided in the form of partial error patterns.

Steps involved in DCR mechanism is described as follows:

i). Let $S_{N}$ be the sender node, $R_{N}$ be the receiver node.

ii). Let $P_{L R}$ and $P_{S R}$ be the long range and short range packets.

iii). Let $Q_{R}$ be the notification that quite a few blocks are needed for recovery. iv). $S_{N}$ collects the packets from upper layer and categorizes as $P_{L R}$ or $\mathrm{P}_{\mathrm{SR}}$. It decides whether to transmit $\mathrm{P}_{\mathrm{LR}}$ or $\mathrm{P}_{\mathrm{SR}}$ using a probability approach and transmit to MAC layer.

If $\mathrm{S}_{\mathrm{N}}$ sends short range packets i.e. $\mathrm{P}_{\mathrm{SR}}$ to the MAC layer, then checksum codes s added at end of each $\mathrm{P}_{\mathrm{SR}}$. Conversely, if $\mathrm{S}_{\mathrm{N}}$ sends long range packets $\left(\mathrm{P}_{\mathrm{LR}}\right)$ to the MAC layer, then FEC codes is added at the end of each $P_{L R}$. Here checksum code will be used by $R_{N}$ in order to verify the packet integrity. Adaptive FEC is used at the receiver end by adding redundant bit for recovering long range of packets without retransmission during LR-SR collisions. This greatly reduces average delay among node to node communication.

\section{LR-SR Collisions:}

DCR can be effortlessly integrated with conventional CSMA protocols with off-the shelf sensor devices. The main contribution includes: Bit error patterns in collided packets is studied and the revelation of long range and short range packet collisions, which enables achieving efficient collision recovery.

A significant performance improvement is achieved by utilizing LRSR collisions under CSMA.

RSSI based error detection mechanism to identify erroneous blocks with almost no overhead.

If $\mathrm{S}_{\mathrm{N}}$
Then
$\mathrm{S}_{\mathrm{N}}$ adds $\mathrm{CRC}$ at the end of each $\mathrm{P}_{\mathrm{SR}}$.
Else
$\mathrm{S}_{\mathrm{N}}$ adds FEC redundant bit at the end of each $\mathrm{P}_{\mathrm{LR}}$
End if

The nodes send their packets to other nodes via CSMA channel. If the receiver nodes receives collided packet then bit error identification pattern is applied. Assume network consists of 7 nodes in total, 6 senders and one receiver. All 6 nodes send their packets to the receiver at the same time using same frequency. If all sender nodes transmit their packets at same time then there exists packet collision. The number of erroneous bits present in long range collided packets can be reduced by adding FEC redundant bits similarly short range collided packets erroneous bits can get reduced by adding checksum codes at the end. Erroneous bits can be identified by applying bit error pattern; therefore the efficiency of receiving packets gets increased.

$\mathrm{S}_{\mathrm{N}}$ approximation performs non-uniform distribution and introduces delay on $\mathrm{P}_{\mathrm{S}}$. The estimation of non-uniform distribution is given by Equation.

$$
i=(t+1) \log x[\eta(x-1)+1]
$$

Where,

$$
=\mathrm{a} \text { random variable with uniform distribution of }
$$
interval $(0,1)$

$\mathrm{x}=10$ for $\mathrm{P}_{\mathrm{LR}}, \mathrm{x}=20$ for $\mathrm{P}_{\mathrm{SR}}$, Delay occurred due to packet collision 
If $\mathrm{S}_{\mathrm{N}}$ senses short packet range then there occurs prior transmission delay of $\mathrm{z}$, where $\mathrm{z}$ represents the time for transmitting packet synchronization header.

Lost packet recovery mechanism:

During transmission packet losses might occur. These packet losses can be recovered using FEC with packet range control method.

i). $P_{\mathrm{SR}}$ lost during LR-SR collisions.

ii). $P_{L R}$ that lacked sufficient blocks (long range packet) to be recovered by the FEC redundant bits.

iii). Losses occurred as a result of other collision types.

Let $\mathrm{C}$ be the erroneous block threshold. When a receiver node $\left(\mathrm{R}_{\mathrm{N}}\right)$ receives a packet, then it verifies whether the received packet is $P_{L R}$ or $\mathrm{P}_{\mathrm{SR}}$. While $\mathrm{R}_{\mathrm{N}}$ starts receiving the packets, the RSSI sampling begins at every ' $z$ ' time units then it is saved in an array. If the sent message is same as the received message then Msg_Ack is sent, else the corrupted block (erroneous block 'B') is computed for identifying erroneous bits.

For corrupted block $\mathrm{B}_{\mathrm{K}}$, if $\mathrm{k}=\mathrm{i} . \mathrm{m}+\mathrm{g}$,

if $\mathrm{g} \neq 0$, then the block can be reconstructed as shown,

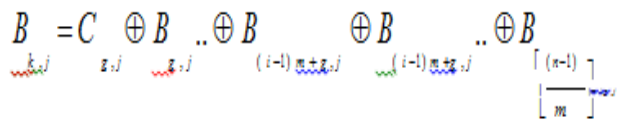

If $\mathrm{g}=0$, then block can be restructured as shown,

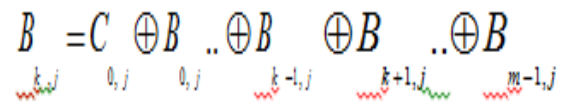

Once the corrupted block is recovered FEC conducts checksum codes and redundant bits and sends an acknowledgement to the $S_{N}$.

\begin{tabular}{l|}
\hline For Short range packet collision: \\
If $R_{N}$ receives $P_{S R}$ \\
Then check RSSI value \\
Apply Checksum code to resolve packet integrity \\
If CRC is valid \\
Then \\
$R_{N}-$ Mack $\rightarrow \mathrm{S}_{\mathrm{N}}$ \\
End if \\
End \\
\end{tabular}

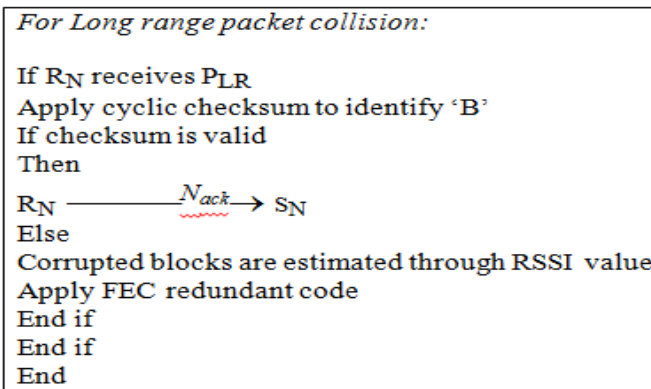

If $\mathrm{S}_{\mathrm{N}}$ sends short range packets, DCR adds a checksum code at the end of each short packet. Later on, receivers use this cyclic checkcode as a checksum to verify the packet integrity. If $S_{N}$ sends long range packets, DCR applied with FEC redundancy codes that allow receivers to recover long packets without retransmission when LRSR-collisions occur. This process reduces communication overhead as well as recovery latency. Long range packet is divided into $n$ blocks, and $m$ redundant blocks is computed that is attached at the end of packets, and hence $\mathrm{R}_{\mathrm{N}}$ can still reconstruct the actual packets that sent by the sender until more than $m$ blocks are corrupted. To compute $n+m$ linear combinations and to accumulate $n+m$ linear combinations to a packet to transmit $\mathrm{n}$ blocks is considered. A few accurate $n$ combinations are adequate for $\mathrm{R}_{\mathrm{N}}$ to restructure the actual data.

During reception of packets at $\mathrm{R}_{\mathrm{N}}$, RSSI values of data or packet is measured. This RSSI value gets increased when collisions occur, but the value remains almost constant if no collision occurs. We conduct experiments to measure the RSSI value in the course of packet reception. Therefore collision block at receiver end $R_{N}$ is estimated through RSSI values (high and low). This is acknowledged through N_Ack. RSSI is measured for every $\delta$ periodic and checks for high or normal then stored in an array. DCR look up the array, if RSSI found to be high then the received packet block is said to be potentially corrupted block.

The total amount of corrupted packets cannot exceed certain level ' $h$ ' and the successful recovery probability ' $R$ ', packet error rate $\mathrm{P}_{\mathrm{pkt}}$, and lower bound $\mathrm{h}$ are all estimated through predicted packet error rate of FEC packets. The average bit error rate in the CSMA channel be $P_{B}$ is given as,

$$
\begin{aligned}
& P \\
& \text { pate }
\end{aligned}=1-(1-P)^{\tau}
$$

The segmentation factor ' $\mathrm{f}$ ' is determined with respect to the packet length (long range or short range). The packet error rate for packet segmentation is given as,

$$
\begin{gathered}
P^{f}=1-(1-P)^{1 / f} \\
p_{\text {bat }}
\end{gathered}
$$

To increase the packet range control the source packet and FEC packets can be adjusted individually. The expected PER of source packets $\mathrm{P}_{\text {src }}$ and expected PER of FEC packets $\mathrm{P}_{\mathrm{FEC}}$ computation is given as,

$$
\begin{aligned}
& P_{\text {src }}=P_{\text {pktin }} \text {, } \\
& P_{F E C}=P_{s k c}^{f r}=\underset{p l s t}{P_{f s}^{*} f t}
\end{aligned}
$$

The proposed scheme chooses the result of lower end to end delay during packet transmission and adaptive FEC with packet range control with minimal bandwidth provides better good-put results.

\section{Simulation Analysis and Results:}

To validate the method proposed here, a simulation scenario with 50 nodes deployed and configured as the subterranean wireless network is used with the specifications mentioned in the table 1 . 
Programming in $\mathrm{C}++$ and Object-oriented Tool Command Language (OTCL) is done to determine the locations of various targeted nodes.

\begin{tabular}{|c|c|}
\hline Parameter & Value \\
\hline Simulation Time & $60 \mathrm{~s}$ \\
\hline Number of Nodes & 100 \\
\hline Routing Protocol & EOR-ACO and CLDHE \\
\hline Simulation Area & $800 \times 800$ \\
\hline Transmission Range & 250 \\
\hline Antenna Type & Omni Antenna \\
\hline $\begin{array}{c}\text { Network Interface } \\
\text { Type }\end{array}$ & Wireless PHY \\
\hline Channel Type & Wireless Channel \\
\hline
\end{tabular}

Table.1: Simulation parameters

The performance of the proposed system CLDHEC is compared with conventional system, which is analyzed in this work using simulation results.

In order to analyze the performances, the packet delivery rate, bandwidth integrity, throughput, delay and leftover energy are compared through simulations

\section{Delivered Packet Rate:}

The rate of packets that sent over to the destination to the rate of packets that sent by the source measures the packet delivery rate. The equation for measuring the rate of packet deliveries is given in equation,

$$
P D R=\frac{\sum P k t s R c v d}{\sum \text { Pkts Sent }}
$$

'Pkts sent' is represented as number of packets sent by the source node and 'Pkts

Received' is represented as number of packets received by the destination for data transmission. The figure shows that the proposed scheme CLDHEC has better delivery rates of packets at the receiver end compared to the existing method OOECS and CARQ_RS.

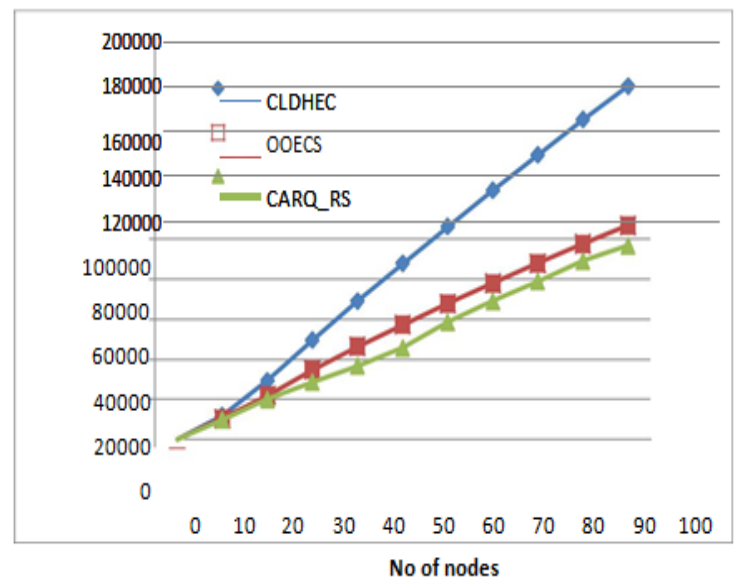

Fig.4: Throughput

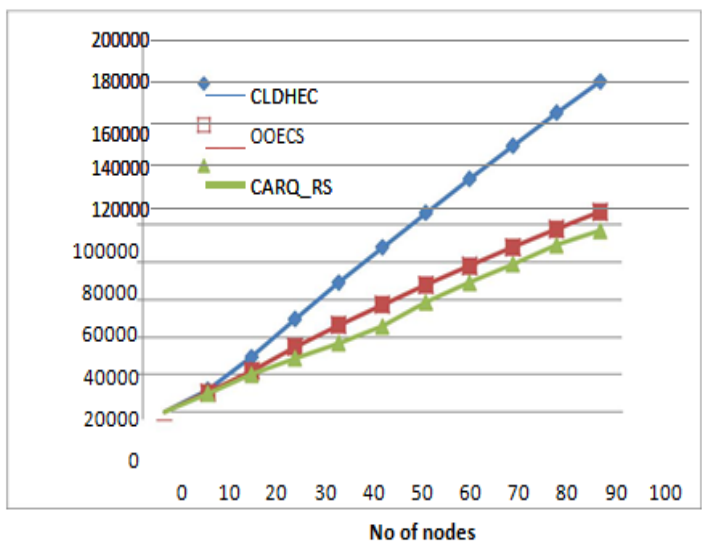

Fig.3: Packet Delivery Rate

\section{Throughput}

Throughput can be defined as the successful transmission of information at the receiver that passed over the network. The overall performance of the network system is measured using the equation

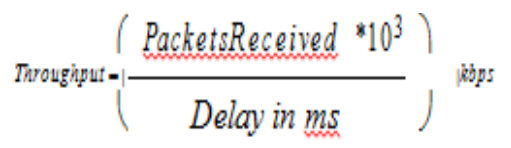

Figure shows that performance of the network throughput which is the comparison of both existing and proposed method. The result proved that the throughput for the proposed scheme CLDHEC is better than the conventional OOECS and CARQ_RS

\section{Packet Loss:}

Packet loss ratio is defined as the ratio of number of packets dropped by the nodes in the network to the total number of packets sent as given in equation,

$$
P D R=\frac{\sum P k \text { lost }}{\sum P k t s \text { Sent }}
$$

Figure shows that packet loss rate that travels from source hub to the destined hub that includes both existing and proposed method. The result proved that the loss or error rate is better for the proposed scheme CLDHEC than the conventional OOECS and CARQ_RS schemes.

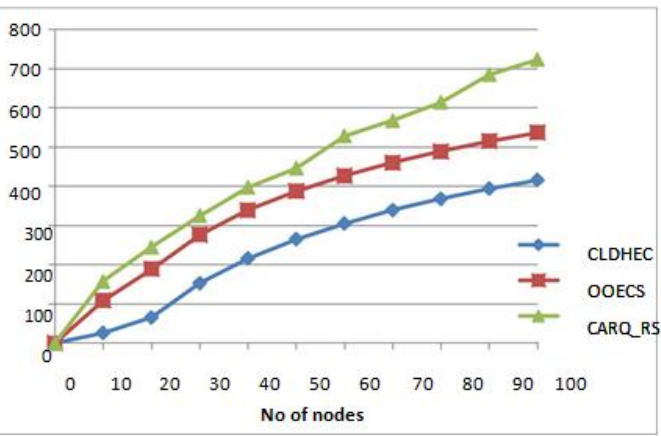

Fig. 5: Packet error rate 


\section{Delay}

Delay is measured for the time difference that takes for the network process to complete their data transmission from one end to the other end. The delay can be measured in terms of time difference exists between the received packets and the sent packets

$$
\text { Delay }=\frac{\sum \text { Rkt Recvd Time }- \text { Pkt Send Time* }}{n}
$$

The delay for the proposed and conventional schemes is shown in the figure. It is clearly shown that the proposed CLDHEC has better performance in terms of ETE-delay compared to the conventional scheme OOECS and CARQ_RS.

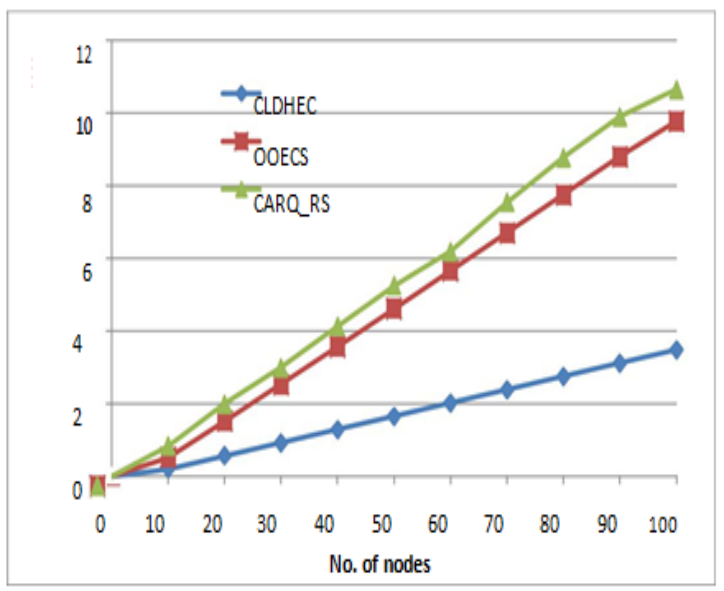

Fig.6: End to End Delay

\section{Conclusions:}

Cross Layer Design based Hybrid Error Control (CLDHEC) is proposed for declining error in WSN. Error control technique such as Adaptive Forward Error Correction with effective packet range control mechanism jointly used to reduce packet collisions over the CSMA channel. Adaptive FEC is used to defend the video transmission by recovering source information losses. Also, FEC mechanism along with packet size control greatly increases the FEC efficiency in wireless networks. If the received packet is corrupted then it is encoded with cyclic checksum codes before retransmission. Packet range control is demonstrated with effective LS-RS collisions that are determined through RSSI values at the receiver end. QoS for different kind of data can be improved by reducing error rate without retransmission using cross layer error control during packet aggregation. Simulation results are analysed and it shows that the proposed scheme results in good throughput and delivery rates with low delay and loss rates.

\section{References:}

[1] Borgonovo F., and Capone A.,(2005) "Efficiency of error-control schemes For real-time wireless applications on The Gilbert channel," IEEE Transactions on Vehicular Technology, Vol. 54, Issue 1, pp. 246-258.
[2] Vuran, M. C., \& Akyildiz, I. F. Sensor networks: A cross layer Transacti on Networking, $17(4)$

(2009). Error control in wireless analysis. IEEE/ACM

[3] Bajic, Ivan V. (2007)"Efficient cross- Layer error control for wireless video multicast." TBC 53, no. 1 :276-285.

[4] Shih, Chi-Huang. (2010)"Adaptive Forward error correction combined with packet size control for wireless video." In Sixth International Conference on Intelligent Information Hiding and Multimedia Signal Processing, pp. 256- 259.

[5] Giorgio Quer, Riccardo Masiero, Michele Rossi and Michele Zorzi,(2009) "Sensing, Compression and Recovery for Wireless Sensor Networks:Monitoring Framework Design",IEEE Global Communication Conference(GLOBECOM), Honululu, HW,

[6] Borgonovo, Flaminio, and Antonio Capone. (2005): "Efficiency of error- Control schemes for real-time wireless applications on the Gilbert channel." IEEE Transactions on Vehicular Technology 54, no. $1246-258$.

[7] Gan, Tong, Lu Gan, and Kai-Kuang Ma. (2007) "Expected runtime distortion based scheduling for scalable video transmission with hybrid FEC/ARQ error control." In Acoustics, Speech and Signal Processing, ICASSP IEEE ternational Conference on, vol. 1, pp.I-829.

[8] Zhai, Fan, Yiftach Eisenberg,Thrasyvoulos N. Pappas, RandallBerry, and AggelosK. Katsaggelos. (2006). "Rate-distortion optimized hybriderror control for real-timepacketized video transmission."IEEE Transactions on ImageProcessing 15, no. 1 40-53.

[9] Chong, Tan, Zou Junni, Wang Min, and Tang Liang.( 2012 ) "Network coding/ARQ hybrid adaptive error- control scheme for video transmission over wireless network." In Control Conference (CCC), 31 st Chinese, pp. 5569-5572

[10] Qaisar, Saad Bin, and Hayder Radha. (2018)"OPERA:An optimal progressive error recovery algorithm for wireless sensor networks." In Sensor, Mesh and Ad Hoc Communications and Networks, 2007. SECON'07. 4th Annual IEEE Communications Society Conference on, pp. 344-352. IEEE, 2007.

[11] Hattori, Shingo, Kentaro Kobayashi, Hiraku Okada, and Masaaki Katayama. (2015): "On- $\quad$ Off Error Control Coding

[12] Scheme for Minimizing Tracking Error in Wireless Feedback Control Systems." IEEE Transactions on Industrial Informatics 11, no. 6 :1411-1421.

[13] Usman, Muhammad, Ning Yang, Mian Ahmad Jan, Xiangjian $\mathrm{He}, \quad$ Min Xu, and Kin-Man Lam. "A joint framework for QoS and QoE for video transmission over wireless multimedia sensor networks." IEEE Transactions on Mobile Computing17, no. 4 746-759.

[14] Marchenko, Nikolaj, and Christian Bettstetter. (2014): "Cooperative ARQ with relay selection: An analytical framework using semi-Markov processes." IEEE transactions on vehicular technology 63, no. 178-190.

[15] Shi, Liqi, and Abraham O. Fapojuwo. (2012): "Crosslayer optimization with minimum power cost in packet wireless sensor networks." Ad no.7:1457-1468. cooperative communication for error rate constrained

Hoc Networks 10, 